\title{
Foreword to the special issue of journal of intelligent manufacturing on uncertain models in intelligent manufacturing systems: dedicated to professor Mistuo Gen for his 70th birthday
}

\author{
Jin Peng • Jinwu Gao
}

Received: 7 November 2014 / Accepted: 4 December 2014 / Published online: 24 December 2014

(C) Springer Science+Business Media New York 2014

It is a real pleasure and honor for us to write this preface to the special issue of Journal of Intelligent Manufacturing (JIM), which focuses on "Uncertain Models in Intelligent Manufacturing Systems". The special issue is comprised of excellent papers selected from IMS2014 conference and marked as a gift in honor and celebration of the 70th birthday of Professor Mitsuo Gen.

Real decisions are usually made in the state of indeterminacy, including randomness and uncertainty. There exist two mathematical systems for modeling indeterminacy. One is probability theory (Kolmogorov 1933) which is the branch of mathematics concerned with probability (interpreted as frequency) and the analysis of random phenomena. The other is uncertainty theory (Liu 2007) which is the branch of mathematics concerned with uncertainty (interpreted as personal belief degree) and the analysis of uncertain phenomena.

Real-world manufacturing systems involve various uncertain factors. In order to improve the efficiency and effectiveness of the manufacturing system in an uncertain environment, we are usually employing various uncertain programming and artificial intelligent techniques. Then we can implement decision support systems or knowledge-based systems by combining the computational results based on mathematical programming or artificial intelligence to simulate, optimize and manage an intelligent manufacturing sys-

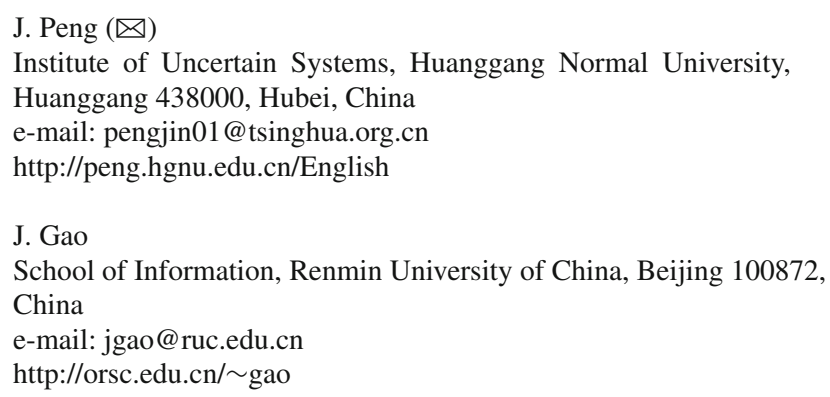

tem. In fact, intelligent manufacturing systems have been one of the most rapidly developing areas in the last decade.

The purpose of this special issue is to provide a channel to guarantee fast publication of extended versions of the highquality conference papers on optimization and management models in intelligent manufacturing systems involving uncertainties. We believe that this special issue will contribute to fostering the development of intelligent manufacturing system in the uncertain environment. In addition, we also hope that the publication of this issue will stimulate further research in the related areas.

This special issue is associated with the Thirteenth International Conference on Information and Management Sciences (IMS2014), sponsored by the International Association for Information and Management Sciences (IMS), which was held at Zhangjiajie, Hunan Province, China during August 3rd to 8th, 2014. Papers for this special issue were solicited not only from participants presented at the IMS2014 Sessions, but also from authors with original high-quality contributions that have neither been published in nor submitted to any journals. Most papers focused on design, planning, modeling, optimization and management of intelligent manufacturing systems.

The conference IMS2014 is aimed to provide a forum to the exchange of ideas on the latest developments in the fields of the applications of information and management sciences, intelligent manufacturing systems among researchers, and to seek opportunities for collaboration among the participants from various countries. In fact, IMS conferences have quickly developed into an important forum for the exchange of new ideas among professionals in the theoretical and practical areas related to information and management sciences.

Founded on July 10, 2005, after over ten years development, the IMS has now more than 50 board members. As the president of IMS Board (2013-2016), Professor Mitsuo Gen 
has made tremendous contributions to this academic organization. He has been invited to give plenary talks many times in this premier international forum for scientists and researchers by presenting state-of-the-art and most recent developments in the area of genetic algorithms and intelligent methods with applications in manufacturing systems design and computers, etc. In addition, he proposed an annual IMS award for best papers rewarding those who have obtained outstanding achievements in scientific research. Owing to Professor Mitsuo Gen's efforts, the excellent papers presented at our conferences can be successfully published in the special issues of famous international journals. Taking the opportunity given by this conference, we are honored to celebrate Professor Mitsuo Gen's 70th birthday.

Dr. Mitsuo Gen has rich academic experience. He received the B.E., M.E., and Ph.D. degrees in electronic engineering from Kogakuin University, Tokyo, Japan, in the years 1969, 1971, and 1975, respectively. Besides, he was granted a Ph.D. degree in informatics from Kyoto University, Kyoto, Japan, in 2006.

Professor Mitsuo Gen was a Senior Research Scientist at Fuzzy Logic Systems Institute, Iizuka, Japan, and a Special Mission Professor at Tokyo University of Science, Tokyo, Japan. He was a Visiting Professor at the Department of Industrial Engineering and Engineering Management at National Tsing Hua University, Hsinchu, Taiwan. He was also Professor Emeritus at the Ashikaga Institute of Technology, Ashikaga, Japan. He was a faculty member at the Ashikaga Institute of Technology, and a professor at the Graduate School of Information, Production and Systems at Waseda University, Kitakyushu, Japan. From 1999 to 2000 he was a visiting professor at the Department of Industrial Engineering and Operations Research at University of California, Berkeley, CA, USA. Likewise at the Department of Industrial Engineering, Texas A \& M University, College Station, TX, USA, in 2000, as well as a Hanyang Chair Professor at Hanyang University, Korea, from 2011 to 2012.

As a leading expert in the domain of evolutionary computing, Professor Mitsuo Gen's research interests cover evolutionary algorithms, manufacturing scheduling, logistics network and decision making. He has authored or coauthored more than 400 journal papers on high level international journals, including IEEE Transaction on Reliability, IEEE Transaction on Automation Sci. Tech., European Journal of Operational Research, Journal of Operational Research Society, OR Spectrum, Networks, Computers \& Operations Research, Computers \& Industrial Engineering, International Journal of Systems Science, Computers in Industry, International Journal of Production Research, International Journal of Production Economics, Journal of Intelligent Manufacturing, Expert Systems with Applications, Soft Computing, Applied Soft Computing, Applied Mathematics \& Computation, etc. His recent citation index and the h-index of his papers reached 15,458 and 55 , respectively. He is currently serving as Editor-in-Chief of Industrial Engineering \& Management Systems, the area editor of Computers \& Industrial Engineering and the associate editor of OR Spectrum. Furthermore he also acts as editorial board member of various international journals including Fuzzy Optimization and Decision Making, International J. of Manufacturing Technology and Management, and Journal of Uncertain Systems.

As a renowned expert acknowledged worldwide, Professor Mitsuo Gen has contributed greatly to the theoretical and experimental genetic algorithms (GA). He has published many books, including Genetic Algorithms and Engineering Design by Wiley in 1997, and Genetic Algorithms and Engineering Optimization by Wiley in 2000, both of which have been translated into Chinese. He is also a coauthor of other books such as Introduction to Evolutionary Algorithms in 2010, Intelligent and Evolutionary Systems in 2009, and Network Models and Optimization: Multiobjective Genetic Algorithm Approach in 2008 by Springer and others. Widely regarded as one of the most influential scientists of our time, Dr. Mitsuo Gen is not only most famous for his GA research, but also he has made contributions to other areas of intelligent systems such as semiconductor manufacturing systems.

Each birthday is a milestone we reach on the road of life. It represents a new beginning and a new chance to get a foothold on the future. Today we take special pride in all the achievements a wonderful person has made. We look forward to another chance to greet Professor Mistuo Gen again and to celebrate his new great work.

This preface is dedicated to Professor Mistuo Gen on the occasion of his 70th birthday. We hope this special issue would bring to Dr. Mitsuo Gen joyful days filled with friendliness, bright days filled with color, and warm days filled with happiness to last throughout all his life!

Besides, we would like to express our sincere thanks to Professor Andrew Kusiak, the editor-in-chief of the Journal of Intelligent Manufacturing, for his encouragement, guidance and support in the preparation of this special volume. Then special thanks gratitude the reviewers who provided the authors with critical, timely and highly constructive feedback. Finally we are also grateful to all the authors who contributed quality refereed papers to this special issue devoted to Professor Gen's 70th birthday. 